\title{
Folding Phenomenon of Major-balance Identities on Restricted Involutions
}

\author{
Tung-Shan Fu, Hsiang-Chun Hsu* and Hsin-Chieh Liao
}

\begin{abstract}
In this paper we prove refined major-balance identities on the 321-avoiding involutions of length $n$, respecting the leading element of permutations. The proof is based on sign-reversing involutions on the lattice paths within a $\lfloor n / 2\rfloor \times\lceil n / 2\rceil$ rectangle. Moreover, we prove affirmatively a question about refined major-balance identities on the 123-avoiding involutions, respecting the number of descents.
\end{abstract}

\section{Introduction}

Let $\mathfrak{S}_{n}$ be the set of permutations of $\{1,2, \ldots, n\}$. A permutation $\sigma=\sigma_{1} \cdots \sigma_{n} \in \mathfrak{S}_{n}$ is called 321-avoiding (123-avoiding, respectively) if it has no decreasing (increasing, respectively) subsequence of length three. Let $\mathfrak{S}_{n}(321)\left(\mathfrak{S}_{n}(123)\right.$, respectively) be the set of 321-avoiding (123-avoiding, respectively) permutations in $\mathfrak{S}_{n}$. It is known that $\left|\mathfrak{S}_{n}(321)\right|=\left|\mathfrak{S}_{n}(123)\right|=C_{n}=\frac{1}{n+1}\left(\begin{array}{c}2 n \\ n\end{array}\right)$, the $n$th Catalan number.

\subsection{Sign-balance for restricted permutations}

The sign-balance of restricted permutations is an interesting theme in enumerative combinatorics. Simion and Schmidt [7] proved the following sign-balance property of $\mathfrak{S}_{n}(321)$ :

$$
\sum_{\sigma \in \mathfrak{S}_{n}(321)}(-1)^{\operatorname{inv}(\sigma)}= \begin{cases}C_{(n-1) / 2} & \text { if } n \text { is odd } \\ 0 & \text { if } n \text { is even }\end{cases}
$$

where $\operatorname{inv}(\sigma)=\#\left\{\left(\sigma_{i}, \sigma_{j}\right): \sigma_{i}>\sigma_{j}\right.$ and $\left.i<j\right\}$ is the inversion number of $\sigma$. Adin and Roichman [1] proved a refinement of this result, respecting the position of the last descent (ldes) of $\sigma$, i.e., $\operatorname{ldes}(\sigma)=\max \left\{i: \sigma_{i}>\sigma_{i+1}\right.$ and $\left.1 \leq i \leq n-1\right\}$.

Theorem 1.1 (Adin-Roichman). For all $n \geq 1$, the following identities hold:

(i) $\sum_{\sigma \in \mathfrak{S}_{2 n+1}(321)}(-1)^{\operatorname{inv}(\sigma)} q^{\operatorname{ldes}(\sigma)}=\sum_{\sigma \in \mathfrak{S}_{n}(321)} q^{2 \cdot \operatorname{ldes}(\sigma)}$,

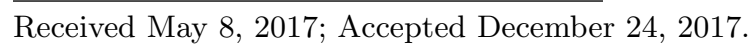

Communicated by Sen-Peng Eu.

2010 Mathematics Subject Classification. 05A05, 05A19.

Key words and phrases. involutions, pattern avoidance, sign-balance, major-balance.

*Corresponding author. 
(ii) $\sum_{\sigma \in \mathfrak{S}_{2 n}(321)}(-1)^{\operatorname{inv}(\sigma)} q^{\operatorname{ldes}(\sigma)}=(1-q) \sum_{\sigma \in \mathfrak{S}_{n}(321)} q^{2 \cdot \operatorname{ldes}(\sigma)}$.

Later, Reifegerste 6] proved an analogous refinement for another permutation statistic, the length of the longest increasing subsequence of the permutations. Eu et al. [4] turned to other families of restricted permutations and obtained refined sign-balance results for 321-avoiding alternating permutations, respecting the leading element and the last element of permutations, respectively.

These results share a folding phenomenon that with respect to a certain statistic the sign-balance generating function for restricted permutations of length $2 n$ essentially equals the ordinary generating function for the permutations of length $n$. Eu et al. [5] described the folding phenomenon in the framework

$$
\sum_{\pi \in \mathscr{X}_{2 n} \text { or }}(-1)^{\operatorname{stat}_{2 n+1}(\pi)} q^{\operatorname{stat}_{2}(\pi)}=f(q) \sum_{\pi \in \mathscr{X}_{n}} q^{2 \cdot \operatorname{stat}_{2}(\pi)},
$$

where $\mathscr{X}_{n}$ is a family of combinatorial objects of size $n$ with statistics stat ${ }_{1}$ and stat $_{2}$, and $f(q)$ is a rational function. In this paper, we present an instance of such a phenomenon on 321-avoiding involutions.

\subsection{Major-balance for 321-avoiding involutions}

The descent set of $\sigma$ is defined as $\operatorname{Des}(\sigma)=\left\{i: \sigma_{i}>\sigma_{i+1}, 1 \leq i \leq n-1\right\}$, and the descent number (des) and major index (maj) of $\sigma$ are defined by

$$
\operatorname{des}(\sigma)=\sum_{i \in \operatorname{Des}(\sigma)} 1 \text { and } \operatorname{maj}(\sigma)=\sum_{i \in \operatorname{Des}(\sigma)} i .
$$

Recall that a permutation $\sigma$ is called an involution if and only if $\sigma^{-1}=\sigma$. Let $\mathscr{I}_{n}(321)$ $\left(\mathscr{I}_{n}(123)\right.$, respectively) be the set of involutions in $\mathfrak{S}_{n}(321)\left(\mathfrak{S}_{n}(123)\right.$, respectively). Simion and Schmidt [7] proved that $\left|\mathscr{I}_{n}(321)\right|=\left|\mathscr{I}_{n}(123)\right|=\left(\begin{array}{c}n \\ \lfloor n / 2\rfloor\end{array}\right)$. Recently, Eu et al. 5] proved the following refined major-balance result on 321-avoiding involutions.

Theorem 1.2 (Eu-Fu-Pan-Ting). For all $n \geq 1$, the following identities hold:

(i) $\sum_{\sigma \in \mathscr{I}_{4 n}(321)}(-1)^{\operatorname{maj}(\sigma)} q^{\operatorname{des}(\sigma)}=\sum_{\sigma \in \mathscr{I}_{2 n}(321)} q^{2 \cdot \operatorname{des}(\sigma)}$,

(ii) $\sum_{\sigma \in \mathscr{I}_{4 n+2}(321)}(-1)^{\operatorname{maj}(\sigma)} q^{\operatorname{des}(\sigma)}=(1-q) \sum_{\sigma \in \mathscr{I}_{2 n}(321)} q^{2 \cdot \operatorname{des}(\sigma)}$,

(iii) $\sum_{\sigma \in \mathscr{I}_{2 n+1}(321)}(-1)^{\operatorname{maj}(\sigma)} q^{\operatorname{des}(\sigma)}=\sum_{\sigma \in \mathscr{I}_{n}(321)} q^{2 \cdot \operatorname{des}(\sigma)}$. 
Meanwhile, they asked a question about an analogous result for 123-avoiding involutions.

Conjecture 1.3 (Eu-Fu-Pan-Ting). For all $n \geq 1$, the following identities hold:

(i) $\sum_{\sigma \in \mathscr{I}_{4 n}(123)}(-1)^{\operatorname{maj}(\sigma)} q^{\operatorname{des}(\sigma)}=q \sum_{\sigma \in \mathscr{I}_{2 n}(123)} q^{2 \cdot \operatorname{des}(\sigma)}$,

(ii) $\sum_{\sigma \in \mathscr{I}_{4 n+2}(123)}(-1)^{\operatorname{maj}(\sigma)} q^{\operatorname{des}(\sigma)}=(1-q) q^{2} \sum_{\sigma \in \mathscr{I}_{2 n}(123)} q^{2 \cdot \operatorname{des}(\sigma)}$,

(iii) $\sum_{\sigma \in \mathscr{I}_{2 n+1}(123)}(-1)^{\operatorname{maj}(\sigma)} q^{\operatorname{des}(\sigma)}=(-1)^{n} q^{2} \sum_{\sigma \in \mathscr{I}_{n}(123)} q^{2 \cdot \operatorname{des}(\sigma)}$.

\subsection{Our work}

Recall that the $q$-binomial coefficients are polynomials defined as

$$
\left[\begin{array}{l}
n \\
k
\end{array}\right]_{q}:=\frac{[n] !_{q}}{[k] !_{q}[n-k] !_{q}}
$$

where $[n] !_{q}=[1]_{q}[2]_{q} \cdots[n]_{q}$ and $[i]_{q}=1+q+\cdots+q^{i-1}$ for any positive integer $i$.

For a permutation $\sigma=\sigma_{1} \cdots \sigma_{n} \in \mathfrak{S}_{n}$, let lead $(\sigma)$ denote the first element of $\sigma$, i.e., $\operatorname{lead}(\sigma)=\sigma_{1}$. In addition to answering the above question, one of the main results in this paper is the following enumeration of joint distributions for two statistics of 321-avoiding involutions.

Theorem 1.4. We have

(i) $\sum_{\substack{\sigma \in \mathscr{I}_{n}(321) \\ \operatorname{des}(\sigma)=k}} q^{\operatorname{maj}(\sigma)}=q^{k^{2}}\left[\begin{array}{c}\lceil n / 2\rceil \\ k\end{array}\right]_{q}\left[\begin{array}{c}\lfloor n / 2\rfloor \\ k\end{array}\right]_{q}$,

(ii) $\sum_{\substack{\sigma \in \mathscr{I}_{n}(321) \\ \operatorname{lead}(\sigma)=\ell}} q^{\operatorname{maj}(\sigma)}=\sum_{k \geq 0} q^{k^{2}+k \ell+\ell-1}\left[\begin{array}{c}\lceil n / 2\rceil-1 \\ k\end{array}\right]_{q}\left[\begin{array}{c}\lfloor n / 2\rfloor-\ell+1 \\ k\end{array}\right]_{q}$.

The proof of the above theorem involves a specialization of the elementary symmetric functions. We remark that the identity in Theorem 1.4(i) has been proved by Barnabei et al. [2] by a different method.

The second main result is the following refined major-balance identities on 321-avoiding involutions, respecting the leading element. A curious point is that the right-hand side of the identity in Theorem 1.5(iv) is a combination of the generating functions of leading element for $\mathscr{I}_{2 n+1}(321)$ and $\mathscr{I}_{2 n}(321)$.

Theorem 1.5. For all $n \geq 1$, we have 
(i) $\sum_{\sigma \in \mathscr{I}_{4 n}(321)}(-1)^{\operatorname{maj}(\sigma)} q^{\operatorname{lead}(\sigma)}=\frac{1}{q} \sum_{\sigma \in \mathscr{I}_{2 n}(321)} q^{2 \cdot \operatorname{lead}(\sigma)}$,

(ii) $\sum_{\sigma \in \mathscr{I}_{4 n+2}(321)}(-1)^{\operatorname{maj}(\sigma)} q^{\operatorname{lead}(\sigma)}=\left(\frac{1}{q}-1\right) \sum_{\sigma \in \mathscr{I}_{2 n+1}(321)} q^{2 \cdot \operatorname{lead}(\sigma)}$,

(iii) $\sum_{\sigma \in \mathscr{I}_{4 n+3}(321)}(-1)^{\operatorname{maj}(\sigma)} q^{\operatorname{lead}(\sigma)}=\left(\frac{2}{q}-1\right) \sum_{\sigma \in \mathscr{I}_{2 n+1}(321)} q^{2 \cdot \operatorname{lead}(\sigma)}$,

(iv) $\sum_{\sigma \in \mathscr{I}_{4 n+1}(321)}(-1)^{\operatorname{maj}(\sigma)} q^{\operatorname{lead}(\sigma)}=\left(\frac{1}{q}-1\right) \sum_{\sigma \in \mathscr{I}_{2 n+1}(321)} q^{2 \cdot \operatorname{lead}(\sigma)}+\sum_{\sigma \in \mathscr{I}_{2 n}(321)} q^{2 \cdot \operatorname{lead}(\sigma)}$.

2. A bijection between 321-avoiding permutations and grand Dyck paths

Let $m, n$ be positive integers. A Dyck path of length $2 n$ is a lattice path from $(0,0)$ to $(n, n)$, using north step $(0,1)$ and east step $(1,0)$, that stays weakly above the line $y=x$. A partial Dyck path of length $n$ is a lattice path from $(0,0)$ to the line $x+y=n$ staying weakly above the line $y=x$. Let $\mathscr{P}_{n}$ be the set of partial Dyck paths of length $n$. Let $\mathscr{B}(n, m)$ denote the set of lattice paths from $(0,0)$ to $(m, n)$ without restriction. Members of $\mathscr{B}(n, m)$ are called grand Dyck paths. Let $\mathrm{N}$ and $\mathrm{E}$ denote a north step and an east step, respectively.

We shall give combinatorial proofs of Theorems 1.4 and 1.5 on the basis of a bijection between $\mathscr{I}_{n}(321)$ and $\mathscr{B}(\lfloor n / 2\rfloor,\lceil n / 2\rceil)$ given by Barnabei et al. [2]. With the partial Dyck paths $\mathscr{P}_{n}$ being the intermediate stage, the bijection is the composition of two maps $\delta: \mathscr{I}_{n}(321) \rightarrow \mathscr{P}_{n}$ and $\xi: \mathscr{P}_{n} \rightarrow \mathscr{B}(\lfloor n / 2\rfloor,\lceil n / 2\rceil)$. First, we describe the map $\delta: \mathscr{I}_{n}(321) \rightarrow \mathscr{P}_{n}$ given by Deutsch et al. 3].

\subsection{The bijection $\delta: \mathscr{I}_{n}(321) \rightarrow \mathscr{P}_{n}$}

Given a permutation $\sigma=\sigma_{1} \cdots \sigma_{n} \in \mathscr{I}_{n}(321)$, we associate $\sigma$ with a path $\tau=\delta(\sigma)=$ $z_{1} \cdots z_{n} \in \mathscr{P}_{n}$, where $z_{i}=\mathrm{N}$ if $\sigma_{i} \geq i$ and $z_{i}=\mathrm{E}$ if $\sigma_{i}<i$.

To find $\delta^{-1}$, we label the steps of $\tau$ from left to right by $1,2, \ldots, n$. Proceeding from right to left across $\tau$, couple each $\mathrm{E}$ step with the nearest uncoupled $\mathrm{N}$ step to its left. Then the cycle structure of the involution $\delta^{-1}(\tau)$ can be determined by taking the labels of a coupled pair as a transposition and an uncoupled $\mathrm{N}$ step as a fixed point. Recall that a permutation is an involution if its cycle structure contains no cycle of length greater than two.

Example 2.1. Consider $\sigma=2136745810911 \in \mathscr{I}_{11}(321)$. The partial Dyck path $\tau=\delta(\sigma)$ is shown on the left-hand side of Figure 2.1. For the inverse map, if we label the 
steps from left to right by $1,2, \ldots, 11$ and traverse $\tau$ backward, then we obtain the cycle structure of $\sigma=\delta^{-1}(\tau)$, i.e., $(12)(3)(46)(57)(8)(910)(11)$.

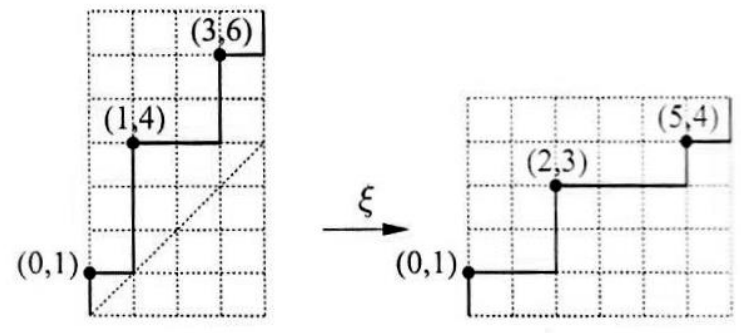

Figure 2.1: An example for the bijection $\xi$ between $\mathscr{P}_{11}$ and $\mathscr{B}(5,6)$

A peak at position $i$ of $\tau$ is an occurrence $z_{i} z_{i+1}=\mathrm{NE}$, which is sometimes identified with the point $p$ between $z_{i}$ and $z_{i+1}$. Note that the coordinate $(x, y)$ of $p$ satisfies $x+y=i$ and that every descent $\sigma_{i}>\sigma_{i+1}$ of $\sigma$ is carried to a peak at position $i$ of $\tau$. We observe that if all fixed points in $\sigma$ are ignored, each east step of $\tau$ must be coupled with a remaining north step to its left. A valley of $\tau$ is an occurrence of EN. A lattice point with coordinate $(x, y)$ is said to be even (odd, respectively) if $x+y$ is even (odd, respectively). For convenience, we say that a peak or valley $p$ is odd (even, respectively) if $p$ is an odd (even, respectively) lattice point. Let $\operatorname{sump}(\tau)$ be the sum of the $x$-coordinates and $y$-coordinates of all peaks in $\tau$.

\subsection{The bijection $\xi: \mathscr{P}_{n} \rightarrow \mathscr{B}(\lfloor n / 2\rfloor,\lceil n / 2\rceil)$}

Let $\tau \in \mathscr{P}_{n}$ be a partial Dyck path with $k$ more $\mathrm{N}$ steps than $\mathrm{E}$ steps $(0 \leq k \leq n)$. Match the $\mathrm{N}$ steps and $\mathrm{E}$ steps that face each other, in the sense that the line segment from the midpoint of $\mathbf{N}$ to the midpoint of $\mathbf{E}$ has slope 1 and stays below the path $\tau$. Then we construct the path $\xi(\tau) \in \mathscr{B}(\lfloor n / 2\rfloor,\lceil n / 2\rceil)$ by changing the first $\lceil k / 2\rceil$ unmatched $\mathrm{N}$ steps into $\mathrm{E}$ steps. Note that a peak $(x, y)$ in $\tau$ is carried to a peak $\left(x^{\prime}, y^{\prime}\right)$ in $\xi(\tau)$ with $x+y=x^{\prime}+y^{\prime}$.

Example 2.2. Following Example 2.1, consider the path $\tau=z_{1} \cdots z_{11} \in \mathscr{P}_{11}$ shown on the left-hand side of Figure 2.1. Note that $\operatorname{sump}(\tau)=15$. The unmatched $\mathrm{N}$ steps are $z_{3}$, $z_{8}$ and $z_{11}$. Then the corresponding path $\xi(\pi) \in \mathscr{B}(5,6)$ is obtained from $\tau$ by changing $z_{3}$ and $z_{8}$ into $\mathrm{E}$ steps, shown on the right-hand side of Figure 2.1. Note that the peaks $(1,4)$ and $(3,6)$ of $\tau$ are carried to the peaks $(2,3)$ and $(5,4)$ of $\xi(\tau)$, respectively and $\operatorname{sump}(\xi(\tau))=15$.

To construct $\xi^{-1}$, given a grand Dyck path $\pi \in \mathscr{B}(\lfloor n / 2\rfloor,\lceil n / 2\rceil)$, match the $\mathrm{N}$ steps and $\mathrm{E}$ steps that face each other in $\pi$. Then the path $\xi^{-1}(\pi)$ is recovered from $\pi$ by 
changing the remaining unmatched $\mathrm{E}$ steps into $\mathrm{N}$ steps.

The following properties about the statistics $\operatorname{maj}(\sigma)$ and $\operatorname{lead}(\sigma)$ hold.

Lemma 2.3. Given a permutation $\sigma \in \mathscr{I}_{n}(321)$ with $\operatorname{lead}(\sigma)=\ell$, let $\tau=\delta(\sigma) \in \mathscr{P}_{n}$ and let $\pi=\xi(\tau) \in \mathscr{B}(\lfloor n / 2\rfloor,\lceil n / 2\rceil)$. Then the following results hold:

(i) $\operatorname{maj}(\sigma)=\operatorname{sump}(\tau)=\operatorname{sump}(\pi)$.

(ii) $1 \leq \ell \leq\lfloor n / 2\rfloor+1$.

(iii) The path $\pi$ passes through the points $(0, \ell-1)$ and $(1, \ell-1)$.

(iv) The number of permutations $\sigma \in \mathscr{I}_{n}(321)$ with $\operatorname{lead}(\sigma)=\ell$ is $\left(\begin{array}{c}n-\ell \\ \lceil n / 2\rceil-1\end{array}\right)$.

Proof. Note that every $i \in \operatorname{Des}(\sigma)$ corresponds to a peak $(x, y)$ of $\tau$ and a peak $\left(x^{\prime}, y^{\prime}\right)$ of $\pi$ with $x+y=x^{\prime}+y^{\prime}=i$. The assertion (i) follows.

Since $\operatorname{lead}(\sigma)=\ell, \sigma_{\ell}=1$. We observe that either $\ell=1$ or $\sigma_{1}<\cdots<\sigma_{\ell-1}$ if $\ell>1$ since $\sigma$ is 321-avoiding. If $\ell>1$ then the entries $2, \ldots, \ell-1$ appear to the right of $\sigma_{\ell}$ and hence $2 \ell-2 \leq n$. Moreover, by the construction of the maps $\delta$ and $\xi$, we observe that the grand Dyck path $\pi$ has the prefix $\mathrm{N}^{\ell-1} \mathrm{E}$. The assertions (ii) and (iii) follow.

Note that the number of permutations $\sigma \in \mathscr{I}_{n}(321)$ with $\operatorname{lead}(\sigma)=\ell$ coincides with the number of lattice paths from the point $(1, \ell-1)$ to the point $(\lceil n / 2\rceil,\lfloor n / 2\rfloor)$. The assertion (iv) follows.

\section{A combinatorial proof of Theorem 1.4}

Define the $k$ th elementary symmetric function in $n$ variables

$$
e_{k}=e_{k}\left(x_{1}, x_{2}, \ldots, x_{n}\right):=\sum_{1 \leq i_{1}<i_{2}<\cdots<i_{k} \leq n} x_{i_{1}} x_{i_{2}} \cdots x_{i_{k}}
$$

Note that $e_{0}\left(x_{1}, \ldots, x_{n}\right)=1$ and $e_{k}\left(x_{1}, \ldots, x_{n}\right)=0$ for $k>n$. Recall the principle specializations of $e_{k}\left(x_{1}, \ldots, x_{n}\right)$ (see e.g., [8, Proposition 7.8.3]):

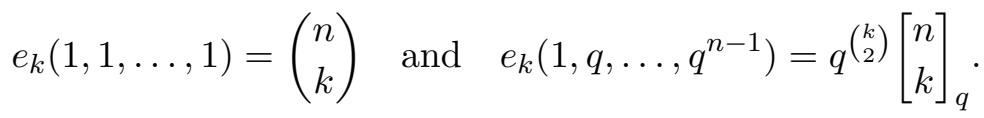

Proof of Theorem 1.4. (i) By the bijection $\xi \circ \delta$ between $\mathscr{I}_{n}(321)$ and $\mathscr{B}(\lfloor n / 2\rfloor,\lceil n / 2\rceil)$, a permutation $\sigma \in \mathscr{I}_{n}(321)$ with $\operatorname{des}(\sigma)=k$ is mapped to a grand Dyck path $\pi=\xi(\delta(\sigma))$ with $k$ peaks, say $\left(x_{1}, y_{1}\right), \ldots,\left(x_{k}, y_{k}\right)$ with $0 \leq x_{1}<\cdots<x_{k} \leq\lceil n / 2\rceil-1$ and $1 \leq y_{1}<$ 
$\cdots<y_{k} \leq\lfloor n / 2\rfloor$. Moreover, $\operatorname{maj}(\sigma)=\operatorname{sump}(\pi)=x_{1}+\cdots+x_{k}+y_{1}+\cdots+y_{k}$. Hence

$$
\begin{aligned}
& \sum_{\substack{\sigma \in \mathscr{I}_{n}(321) \\
\operatorname{des}(\sigma)=k}} q^{\operatorname{maj}(\sigma)}=\sum_{\substack{0 \leq x_{1}<\cdots<x_{k} \leq\lceil n / 2\rceil-1 \\
1 \leq y_{1}<\cdots<y_{k} \leq\lfloor n / 2\rfloor}} q^{x_{1}+\cdots+x_{k}} \cdot q^{y_{1}+\cdots+y_{k}} \\
& =e_{k}\left(1, q, \ldots, q^{\lceil n / 2\rceil-1}\right) \cdot e_{k}\left(q, q^{2}, \ldots, q^{\lceil n / 2\rceil}\right) \\
& =q^{k^{2}}\left[\begin{array}{c}
\lceil n / 2\rceil \\
k
\end{array}\right]_{q}\left[\begin{array}{c}
\lfloor n / 2\rfloor \\
k
\end{array}\right]_{q} .
\end{aligned}
$$

(ii) Let $\sigma \in \mathscr{I}_{n}(321)$ be a permutation with $\operatorname{lead}(\sigma)=\ell$ and $\operatorname{des}(\sigma)=k$. Then the corresponding grand Dyck path $\pi=\xi(\delta(\sigma))$ can be factorized as $\pi=\mathrm{N}^{\ell-1} \mathrm{E} \mu$. If $\ell=1$ then the segment $\mu$ contains $k$ peaks, say $\left(x_{1}, y_{1}\right), \ldots,\left(x_{k}, y_{k}\right)$ with $1 \leq x_{1}<\cdots<x_{k} \leq$ $\lceil n / 2\rceil-1$ and $1 \leq y_{1}<\cdots<y_{k} \leq\lfloor n / 2\rfloor$. Hence

$$
\begin{aligned}
\sum_{\substack{\sigma \in \mathscr{I}_{n}(321) \\
\operatorname{des}(\sigma)=k \\
\operatorname{lead}(\sigma)=1}} q^{\operatorname{maj}(\sigma)} & =e_{k}\left(q, q^{2}, \ldots, q^{\lceil n / 2\rceil-1}\right) \cdot e_{k}\left(q, q^{2}, \ldots, q^{\lceil n / 2\rceil}\right) \\
& =q^{k^{2}+k}\left[\begin{array}{c}
\lceil n / 2\rceil-1 \\
k
\end{array}\right]_{q}\left[\begin{array}{c}
\lfloor n / 2\rfloor \\
k
\end{array}\right]_{q} .
\end{aligned}
$$

Otherwise $\ell>1$, and the segment $\mu$ contains another $k-1$ peaks, say $\left(x_{1}, y_{1}\right), \ldots,\left(x_{k-1}\right.$, $\left.y_{k-1}\right)$ with $1 \leq x_{1}<\cdots<x_{k-1} \leq\lceil n / 2\rceil-1$ and $\ell \leq y_{1}<\cdots<y_{k-1} \leq\lfloor n / 2\rfloor$. Hence

$$
\begin{aligned}
& \sum_{\substack{\sigma \in \mathscr{I}_{n}(321) \\
\operatorname{des}(\sigma)=k \\
\operatorname{lead}(\sigma)=\ell}} q^{\operatorname{maj}(\sigma)}=q^{\ell-1} e_{k-1}\left(q, q^{2}, \ldots, q^{\lceil n / 2\rceil-1}\right) e_{k-1}\left(q^{\ell}, q^{\ell+1}, \ldots, q^{\lfloor n / 2\rfloor}\right) \\
& =q^{(k-1)^{2}+(k-1) \ell+\ell-1}\left[\begin{array}{c}
\lceil n / 2\rceil-1 \\
k-1
\end{array}\right]_{q}\left[\begin{array}{c}
\lfloor n / 2\rfloor-\ell+1 \\
k-1
\end{array}\right]_{q} .
\end{aligned}
$$

The assertion follows.

We remark that Barnabei et al. 22 proved Theorem 1.4(i) by establishing a bijection between the paths in $\mathscr{B}(\lfloor n / 2\rfloor,\lceil n / 2\rceil)$ and the partitions whose Young diagrams fit inside the $\lfloor n / 2\rfloor \times\lceil n / 2\rceil$-rectangle so that the descent set of $\sigma \in \mathscr{I}_{n}(321)$ is carried to the hook-decomposition of the mapped partition.

With the result in Theorem 1.4(ii), we give an arithmetic verification of Theorem 1.5 as follows. For positive integers $m, n$, we have the following facts (i) $[m]_{q=-1}=0$ if and only if $m$ is even, and (ii) if $m, n$ have the same parity, then

$$
\lim _{q \rightarrow-1} \frac{[n]_{q}}{[m]_{q}}= \begin{cases}\frac{n}{m} & \text { if } m, n \text { are even } \\ 1 & \text { if } m, n \text { are odd. }\end{cases}
$$


Making use of the above facts, we observe that

$$
\begin{aligned}
{\left[\begin{array}{l}
n \\
k
\end{array}\right]_{q=-1} } & =\lim _{q \rightarrow-1} \frac{[n]_{q}[n-1]_{q} \cdots[n-k+1]_{q}}{[1]_{q}[2]_{q} \cdots[k]_{q}} \\
& = \begin{cases}0 & \text { if } n \text { is even and } k \text { is odd, } \\
\left(\begin{array}{ll}
\lfloor n / 2\rfloor \\
\lfloor k / 2\rfloor
\end{array}\right) & \text { otherwise. }\end{cases}
\end{aligned}
$$

Now, we verify the identity in Theorem 1.5(i). By Theorem 1.4(ii), we have the lefthand side

$$
\sum_{\substack{\sigma \in \mathscr{I}_{4 n}(321) \\
\operatorname{lead}(\sigma)=\ell}}(-1)^{\operatorname{maj}(\sigma)}=\lim _{q \rightarrow-1} \sum_{k \geq 0} q^{k^{2}+k \ell+\ell-1}\left[\begin{array}{c}
2 n-1 \\
k
\end{array}\right]_{q}\left[\begin{array}{c}
2 n-\ell+1 \\
k
\end{array}\right]_{q} .
$$

If $\ell$ is odd, say $\ell=2 \ell^{\prime}-1$ then by (3.1) we have

$$
\begin{aligned}
\sum_{\substack{\sigma \in \mathscr{I}_{4 n}(321) \\
\operatorname{lead}(\sigma)=2 \ell^{\prime}-1}}(-1)^{\operatorname{maj}(\sigma)} & =\sum_{k^{\prime} \geq 0}\left(\begin{array}{c}
n-1 \\
k^{\prime}
\end{array}\right)\left(\begin{array}{c}
n-\ell^{\prime}+1 \\
k^{\prime}
\end{array}\right) \\
& =\left(\begin{array}{c}
2 n-\ell^{\prime} \\
n-1
\end{array}\right)=\left|\left\{\sigma \in \mathscr{I}_{2 n}(321): \operatorname{lead}(\sigma)=\ell^{\prime}\right\}\right| .
\end{aligned}
$$

If $\ell$ is even, say $\ell=2 \ell^{\prime}$, then

$$
\begin{aligned}
\sum_{\substack{\sigma \in \mathscr{I}_{4 n}(321) \\
\operatorname{lead}(\sigma)=2 \ell^{\prime}}}(-1)^{\operatorname{maj}(\sigma)} & =\sum_{k \geq 0}(-1)^{k^{2}-1}\left[\begin{array}{c}
2 n-1 \\
k
\end{array}\right]_{q=-1}\left[\begin{array}{c}
2 n-2 \ell^{\prime}+1 \\
k
\end{array}\right]_{q=-1} \\
& =\sum_{k=0}^{2 n-2 \ell^{\prime}+1}(-1)^{k^{2}-1}\left(\begin{array}{l}
n-1 \\
\lfloor k / 2\rfloor
\end{array}\right)\left(\begin{array}{l}
n-\ell^{\prime} \\
\lfloor k / 2\rfloor
\end{array}\right) \\
& =0 .
\end{aligned}
$$

This agrees with the right-hand side of Theorem 1.5(i). The other identities (ii), (iii) and (iv) of Theorem 1.5 can be verified in a similar manner.

\section{A combinatorial proof of Theorem 1.5}

For any grand Dyck path $\pi \in \mathscr{B}(n, m)$, we factorize $\pi$ as $\pi=\mu_{0} \mu_{1} \cdots \mu_{d}$, where each segment $\mu_{2 i}\left(\mu_{2 i+1}\right.$, respectively) is a maximal sequence of consecutive $N$ steps (E steps, respectively). This is called the primal factorization of $\pi$. Note that $\mu_{0}$ is empty if $\pi$ starts with an east step.

According to the length of $\mu_{0}$, we partition the set $\mathscr{B}(n, m)$ into subsets $\mathscr{B}_{j}(n, m)$ for $0 \leq j \leq n$, where $\mathscr{B}_{j}(n, m)$ consists of the paths passing the points $(0, j)$ and $(1, j)$. By Lemma 2.3(iii), we have the following result. 
Lemma 4.1. For $0 \leq j \leq\lfloor n / 2\rfloor$, the paths in $\mathscr{B}_{j}(\lfloor n / 2\rfloor,\lceil n / 2\rceil)$ are in one-to-one correspondence with the permutations $\sigma \in \mathscr{I}_{n}(321)$ with $\operatorname{lead}(\sigma)=j+1$.

\subsection{The case $\Phi_{1}: \mathscr{B}(2 n, 2 n) \rightarrow \mathscr{B}(2 n, 2 n)$}

To prove Theorem 1.5(i), we shall establish a sump-parity-reversing involution $\Phi_{1}: \mathscr{B}(2 n$, $2 n) \rightarrow \mathscr{B}(2 n, 2 n)$ while preserving the initial segment from the beginning to the first east step. Let $\mathscr{F}(2 n, 2 n) \subseteq \mathscr{B}(2 n, 2 n)$ be the set of fixed points of the map $\Phi_{1}$. The set $\mathscr{F}(2 n, 2 n)$ can be constructed from $\mathscr{B}(n, n)$ as follows.

For each path $\omega \in \mathscr{B}(n, n)$, we form a path $\gamma(\omega)$ by duplicating every step of $\omega$. Then $\gamma(\omega) \in \mathscr{B}(2 n, 2 n)$. Note that the peaks and valleys of $\gamma(\omega)$ are all even lattice points. Moreover, every path without odd peaks and odd valleys in $\mathscr{B}(2 n, 2 n)$ can be reduced to a path in $\mathscr{B}(n, n)$ by the reverse operation. For example, for $\omega=\operatorname{NEENEN} \in \mathscr{B}(3,3)$, the path $\gamma(\omega)$ is shown as Figure 4.1 .

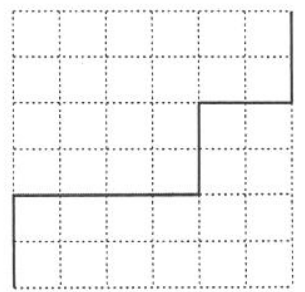

Figure 4.1: Construction of the path $\gamma(\omega)$ for $\omega=\operatorname{NEENEN~} \in \mathscr{B}(3,3)$

The set $\mathscr{F}(2 n, 2 n)$ is defined by

$$
\mathscr{F}(2 n, 2 n)=\{\gamma(\omega): \omega \in \mathscr{B}(n, n)\}
$$

and for $0 \leq i \leq 2 n$, the subset $\mathscr{F}_{i}(2 n, 2 n)$ is defined by

$$
\mathscr{F}_{i}(2 n, 2 n)=\mathscr{F}(2 n, 2 n) \cap \mathscr{B}_{i}(2 n, 2 n) .
$$

Note that $\mathscr{F}_{i}(2 n, 2 n)$ is empty if $i$ is odd. We have the following immediate observation.

Lemma 4.2. For $0 \leq j \leq n$ and any path $\omega \in \mathscr{B}_{j}(n, n)$, the following properties hold:

(i) $\gamma(\omega) \in \mathscr{F}_{2 j}(2 n, 2 n)$ and $\operatorname{sump}(\gamma(\omega))=2 \cdot \operatorname{sump}(\omega)$.

(ii) $\left|\mathscr{F}_{2 j}(2 n, 2 n)\right|=\left|\mathscr{B}_{j}(n, n)\right|$ and $\left|\mathscr{F}_{2 j+1}(2 n, 2 n)\right|=0$.

(iii) The set $\mathscr{F}(2 n, 2 n)$ consists of all the paths without odd peaks and odd valleys in $\mathscr{B}(2 n, 2 n)$. 
Now, we construct the involution $\Phi_{1}$ on $\mathscr{B}(2 n, 2 n)$.

Algorithm 4.3. Given a path $\pi \in \mathscr{B}(2 n, 2 n)$, let $\pi=\mu_{0} \mu_{1} \cdots \mu_{d}$ be the primal factorization of $\pi$. If every segment $\mu_{i}$ contains an even number of steps then $\Phi_{1}(\pi)=\pi$. Otherwise, find the greatest integer $k$ such that $\mu_{k}$ contains an odd number of steps. The path $\Phi_{1}(\pi)$ is obtained from $\pi$ by interchanging the first step of $\mu_{k}$ and the last step of $\mu_{k-1}$.

Example 4.4. Let $\pi$ be the path shown on the left-hand side of Figure 4.2, with the primal factorization $\pi=\mu_{0} \mu_{1} \cdots \mu_{5}$. Then $\mu_{4}=$ NNN is the last segment of odd length. Hence $\Phi_{1}(\pi)$ is obtained from $\pi$ by interchanging the first step of $\mu_{4}$ and the last step of $\mu_{3}$, shown on the right-hand side of Figure 4.2 .
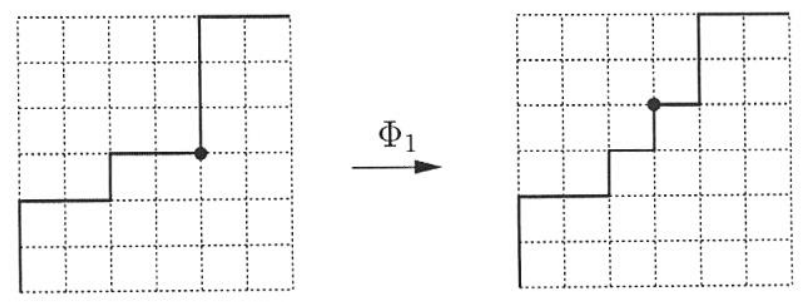

Figure 4.2: An example for sump-parity-reversing involution $\Phi_{1}$ on grand Dyck paths

Lemma 4.5. For the primal factorization $\pi=\mu_{0} \mu_{1} \cdots \mu_{d}$ of a path $\pi \in \mathscr{B}(2 n, 2 n)$, if $k$ is the greatest integer such that $\mu_{k}$ contains an odd number of steps then $k \neq 0$ and $k \neq 1$, i.e., $\Phi_{1}(\pi)$ preserves the first segment $\mu_{0}$ of $\pi$.

Proof. The assertion follows from the fact that $\pi$ has $2 n$ east steps and $2 n$ north steps.

Proposition 4.6. For $0 \leq i \leq 2 n$, the map $\Phi_{1}$ establishes a refined involution on $\mathscr{B}_{i}(2 n, 2 n)-\mathscr{F}_{i}(2 n, 2 n)$. Furthermore, a path $\pi$ is carried to a path $\Phi_{1}(\pi)$ such that $\operatorname{sump}\left(\Phi_{1}(\pi)\right)$ has the opposite parity of $\operatorname{sump}(\pi)$.

Proof. Given a path $\pi \in \mathscr{B}(2 n, 2 n)$, suppose $\Phi_{1}(\pi) \neq \pi$. By Lemma $4.2, \pi$ contains a peak (or valley) which is an odd lattice point. By Algorithm 4.3, we find the last odd peak (or valley) $p$. The path $\Phi(\pi)$ is obtained by interchanging the $\mathrm{N}$ and $\mathrm{E}$ steps adjacent at $p$. This changes the last odd peak (or valley) of $\pi$ into the last odd valley (or peak) of $\Phi(\pi)$. Moreover, by Lemma 4.5, $\Phi_{1}$ is an involution, restricted to each subset $\mathscr{B}_{i}(2 n, 2 n)-\mathscr{F}_{i}(2 n, 2 n)$. We observe that there is exactly one odd lattice point affected. Hence $\operatorname{sump}\left(\Phi_{1}(\pi)\right)$ has the opposite parity of $\operatorname{sump}(\pi)$. 
Proof of Theorem 1.5(i).

$$
\begin{aligned}
\sum_{\sigma \in \mathscr{I}_{4 n}(321)}(-1)^{\operatorname{maj}(\sigma)} q^{\operatorname{lead}(\sigma)} & =\sum_{i=0}^{2 n}\left(\sum_{\pi \in \mathscr{B}_{i}(2 n, 2 n)}(-1)^{\operatorname{sump}(\pi)}\right) q^{i+1} \\
& =\sum_{j=0}^{n}\left(\sum_{\pi \in \mathscr{F}_{2 j}(2 n, 2 n)} q^{2 j+1}\right) \\
& =\sum_{j=0}^{n}\left|\mathscr{B}_{j}(n, n)\right| q^{2 j+1} \\
& =\frac{1}{q} \sum_{\sigma \in \mathscr{I}_{2 n}(321)} q^{2 \cdot \operatorname{lead}(\sigma)} .
\end{aligned}
$$

4.2. The case $\Phi_{2}: \mathscr{B}(2 n+1,2 n+1) \rightarrow \mathscr{B}(2 n+1,2 n+1)$

To prove Theorem 1.5(ii), we shall establish a sump-parity-reversing involution $\Phi_{2}: \mathscr{B}_{i}(2 n$ $+1,2 n+1) \rightarrow \mathscr{B}_{i}(2 n+1,2 n+1)$ for $0 \leq i \leq 2 n+1$. Let $\mathscr{F}(2 n+1,2 n+1) \subseteq \mathscr{B}(2 n+1,2 n+1)$ be the set of fixed points of the map $\Phi_{2}$. For $0 \leq i \leq 2 n+1$, we define

$$
\mathscr{F}_{i}(2 n+1,2 n+1)=\mathscr{F}(2 n+1,2 n+1) \cap \mathscr{B}_{i}(2 n+1,2 n+1) .
$$

The set $\mathscr{F}(2 n+1,2 n+1)$ can be constructed from $\mathscr{B}(n, n+1)$ as follows. For each path $\omega \in \mathscr{B}_{j}(n, n+1)(0 \leq j \leq n)$, we form a path $\gamma(\omega)$ by duplicating every step of $\omega$. Note that $\gamma(\omega)$ is from $(0,0)$ to $(2 n+2,2 n)$ with the prefix $\mathrm{N}^{2 j}$ EE. Factorize $\gamma(\omega)$ as $\gamma(\omega)=\mathrm{N}^{2 j} \mathrm{EE} \beta$. Then we create two paths $\phi_{1}(\omega), \phi_{2}(\omega) \in \mathscr{B}(2 n+1,2 n+1)$ from $\gamma(\omega)$ by

$$
\phi_{1}(\omega)=\mathrm{N}^{2 j} \mathrm{EN} \beta, \quad \phi_{2}(\omega)=\mathrm{N}^{2 j+1} \mathrm{E} \beta,
$$

i.e., $\phi_{1}(\omega)\left(\phi_{2}(\omega)\right.$, respectively) is obtained from $\gamma(\omega)$ by changing the second (first, respectively) east step into a north step. Note that the segment $\beta$ of $\phi_{1}(\omega), \phi_{2}(\omega)$ goes from $(1,2 j+1)$ to $(2 n+1,2 n+1)$ and that every segment in the primal factorization of $\beta$ contains an even number of steps. For example, let $\omega=\operatorname{NEENE} \in \mathscr{B}_{1}(2,3)$. Then the path $\gamma(\omega)$ is shown on the left-hand side of Figure 4.3 and the paths $\phi_{1}(\omega), \phi_{2}(\omega)$ are shown on the right-hand side of Figure 4.3 .
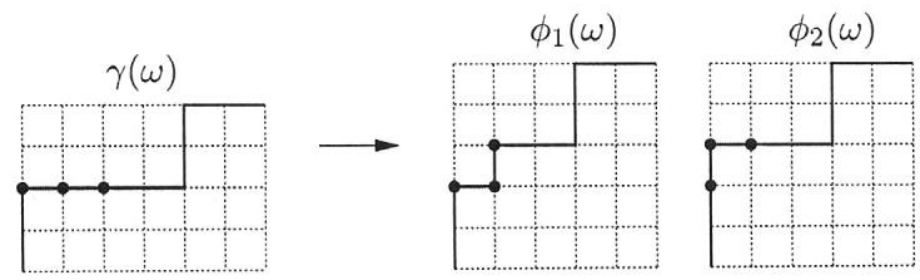

Figure 4.3: Construction of the paths $\phi_{1}(\omega)$ and $\phi_{2}(\omega)$ for $\omega=$ NEENE 
For $0 \leq j \leq n$, define

$$
\begin{aligned}
\mathscr{F}_{2 j}(2 n+1,2 n+1) & =\left\{\phi_{1}(\omega): \omega \in \mathscr{B}_{j}(n, n+1)\right\}, \\
\mathscr{F}_{2 j+1}(2 n+1,2 n+1) & =\left\{\phi_{2}(\omega): \omega \in \mathscr{B}_{j}(n, n+1)\right\} .
\end{aligned}
$$

We have the following immediate observation.

Lemma 4.7. For $0 \leq j \leq n$ and any path $\omega \in \mathscr{B}_{j}(n, n+1)$, the following properties hold:

(i) The path $\phi_{1}(\omega) \in \mathscr{F}_{2 j}(2 n+1,2 n+1)$ contains a unique odd valley $(1,2 j)$ and no odd peaks.

(ii) The path $\phi_{2}(\omega) \in \mathscr{F}_{2 j+1}(2 n+1,2 n+1)$ contains a unique odd peak $(0,2 j+1)$ and no odd valleys.

(iii) $\left|\mathscr{F}_{2 j}(2 n+1,2 n+1)\right|=\left|\mathscr{F}_{2 j+1}(2 n+1,2 n+1)\right|=\left|\mathscr{B}_{j}(n, n+1)\right|$.

It follows that $\operatorname{sump}\left(\phi_{1}(\omega)\right)$ is even and $\operatorname{sump}\left(\phi_{2}(\omega)\right)$ is odd. Note that every path $\pi \in \mathscr{B}(2 n+1,2 n+1)$ contains at least one odd valley or odd peak since $\pi$ has $2 n+1$ east steps and $2 n+1$ north steps. In fact, the set $\mathscr{F}(2 n+1,2 n+1)$ consists of all the paths in $\mathscr{B}(2 n+1,2 n+1)$ either containing a unique odd valley in the line $x=1$ and no odd peaks, or containing a unique odd peak in the line $x=0$ and no odd valleys.

Now, we construct the involution $\Phi_{2}$ on $\mathscr{B}(2 n+1,2 n+1)$.

Algorithm 4.8. Given a path $\pi \in \mathscr{B}(2 n+1,2 n+1)$, let $\mu_{0} \mu_{1} \cdots \mu_{d}$ be the primal factorization of $\pi$. According to the parity of the length of $\mu_{0}$, there are two cases.

Case 1. $\mu_{0}$ is odd, say $\mu_{0}=N^{2 j+1}$. Find the greatest integer $k \geq 1$ such that $\mu_{k}$ contains an odd number of steps. If $k=1$ then let $\Phi_{2}(\pi)=\pi$. Otherwise, the path $\Phi_{1}(\pi)$ is obtained from $\pi$ by interchanging the first step of $\mu_{k}$ and the last step of $\mu_{k-1}$.

Case 2. $\mu_{0}$ is even, say $\mu_{0}=N^{2 j}$. If $\mu_{1}=E$ and $\mu_{2}$ is the only other segment containing an odd number of steps (i.e., $\mu_{t}$ is of even length for all $t \geq 3$ ) then let $\Phi_{2}(\pi)=\pi$. Otherwise, find the greatest integer $k \geq 3$ such that $\mu_{k}$ contains an odd number of steps. Then the path $\Phi_{2}(\pi)$ is obtained from $\pi$ by interchanging the first step of $\mu_{k}$ and the last step of $\mu_{k-1}$.

It is obvious that the map $\Phi_{2}$ preserves the segment $\mu_{0}$, i.e., $\Phi_{2}$ is a map restricted to each subset $\mathscr{B}_{i}(2 n+1,2 n+1)$ for $0 \leq i \leq 2 n+1$.

Example 4.9. Let $\pi$ be the path shown on the left-hand side of Figure 4.4, with the primal factorization $\pi=\mu_{0} \mu_{1} \cdots \mu_{7}$. Then $\mu_{5}=$ EEE is the last segment of odd length. Hence $\Phi_{2}(\pi)$ is obtained from $\pi$ by interchanging the first step of $\mu_{5}$ and the last step of $\mu_{4}$, shown on the right-hand side of Figure 4.4 . 

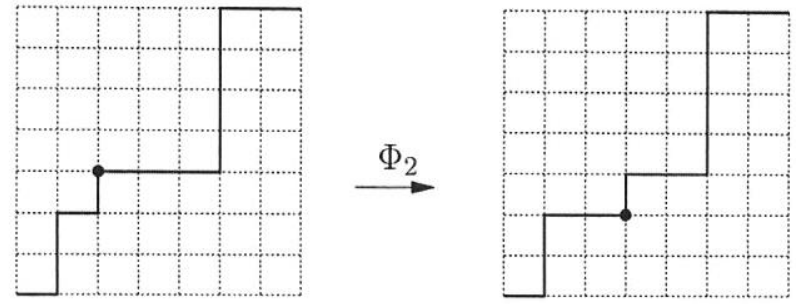

Figure 4.4: An example for the map $\Phi_{2}: \mathscr{B}(7,7) \rightarrow \mathscr{B}(7,7)$

Proposition 4.10. For $0 \leq i \leq 2 n+1$, the map $\Phi_{2}$ establishes a refined involution on $\mathscr{B}_{i}(2 n+1,2 n+1)-\mathscr{F}_{i}(2 n+1,2 n+1)$. Moreover, a path $\pi$ is carried to a path $\Phi_{2}(\pi)$ such that $\operatorname{sump}\left(\Phi_{2}(\pi)\right)$ has the opposite parity of $\operatorname{sump}(\pi)$.

Proof. Given a path $\pi \in \mathscr{B}(2 n+1,2 n+1)$, suppose $\Phi_{2}(\pi) \neq \pi$. By Lemma 4.7, $\pi$ either contains an odd peak $(x, y)$ with $x>0$ or contains an odd valley $\left(x^{\prime}, y^{\prime}\right)$ with $x^{\prime}>1$. By Algorithm 4.8, we find the last odd peak (or valley) $p$ and construct the path $\Phi_{2}(\pi)$ by interchanging the $\mathrm{N}$ and $\mathrm{E}$ steps adjacent at $p$. By the same argument as in the proof of Proposition 4.6, the assertion is proved.

Proof of Theorem 1.5(ii).

$$
\begin{aligned}
& \sum_{\sigma \in \mathscr{I}_{4 n+2}(321)}(-1)^{\operatorname{maj}(\sigma)} q^{\operatorname{lead}(\sigma)} \\
= & \sum_{i=0}^{2 n+1}\left(\sum_{\pi \in \mathscr{B}_{i}(2 n+1,2 n+1)}(-1)^{\operatorname{sump}(\pi)}\right) q^{i+1} \\
= & \sum_{j=0}^{n}\left(\sum_{\pi \in \mathscr{F}_{2 j}(2 n+1,2 n+1)} q^{2 j+1}-\sum_{\pi \in \mathscr{F}_{2 j+1}(2 n+1,2 n+1)} q^{2 j+2}\right) \\
= & \sum_{j=0}^{n}\left|\mathscr{B}_{j}(n, n+1)\right| q^{2 j+1}-\sum_{j=0}^{n}\left|\mathscr{B}_{j}(n, n+1)\right| q^{2 j+2} \\
= & \left(\frac{1}{q}-1\right) \sum_{\sigma \in \mathscr{I}_{2 n+1}(321)} q^{2 \cdot \operatorname{lead}(\sigma)} .
\end{aligned}
$$

4.3. The case $\Phi_{3}: \mathscr{B}(2 n+1,2 n+2) \rightarrow \mathscr{B}(2 n+1,2 n+2)$

To prove Theorem 1.5(iii), we shall establish a sump-parity-reversing involution $\Phi_{3}: \mathscr{B}_{i}(2 n$ $+1,2 n+2) \rightarrow \mathscr{B}_{i}(2 n+1,2 n+2)$ for $0 \leq i \leq 2 n+1$. Let $\mathscr{F}(2 n+1,2 n+2) \subseteq \mathscr{B}(2 n+1,2 n+2)$ be the set of fixed points of the map $\Phi_{3}$. For $0 \leq i \leq 2 n+1$, we define

$$
\mathscr{F}_{i}(2 n+1,2 n+2)=\mathscr{F}(2 n+1,2 n+2) \cap \mathscr{B}_{i}(2 n+1,2 n+2) .
$$


The set $\mathscr{F}(2 n+1,2 n+2)$ can be constructed from $\mathscr{B}(n, n+1)$ as follows. For each path $\omega \in \mathscr{B}_{j}(n, n+1)(0 \leq j \leq n)$, we form a path $\gamma(\omega)$ by duplicating every step of $\omega$. Note that $\gamma(\omega)$ is from $(0,0)$ to $(2 n+2,2 n)$ with the prefix $\mathrm{N}^{2 j}$ EE. Factorize $\gamma(\omega)$ as $\gamma(\omega)=\mathrm{N}^{2 j} \mathrm{EE} \beta$. Then we create three paths $\psi_{0}(\omega), \psi_{1}(\omega), \psi_{2}(\omega) \in \mathscr{B}(2 n+1,2 n+2)$ from $\gamma(\omega)$ by

$$
\psi_{0}(\omega)=\gamma(\omega) \mathrm{N}, \quad \psi_{1}(\omega)=\mathrm{N}^{2 j} \mathrm{EN} \beta \mathrm{E}, \quad \psi_{2}(\omega)=\mathrm{N}^{2 j+1} \mathrm{E} \beta \mathrm{E} .
$$

Note that $\psi_{0}(\omega)$ is obtained from $\gamma(\omega)$ by appending a north step in the end and that $\psi_{1}(\omega)\left(\psi_{2}(\omega)\right.$, respectively) is obtained from $\gamma(\omega)$ inserting a north step after (before, respectively) the first east step and moving the second east step to the end. For example, for $\omega=$ NEENE $\in \mathscr{B}_{1}(2,3)$, the paths $\psi_{0}(\omega), \psi_{1}(\omega)$ and $\psi_{2}(\omega)$ are shown in Figure 4.5 .

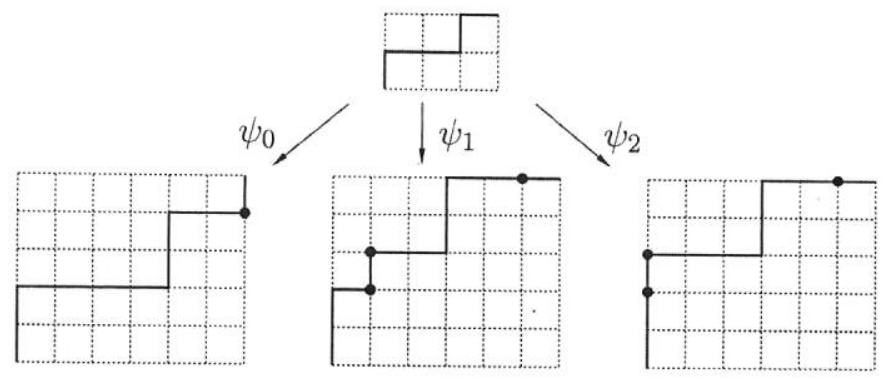

Figure 4.5: Construction of the paths $\psi_{0}(\omega), \psi_{1}(\omega)$ and $\psi_{2}(\omega)$ for $\omega=$ NEENE

We define the refinement of the set $\mathscr{F}(2 n+1,2 n+2)$. For $0 \leq j \leq n$, let

$$
\begin{aligned}
\mathscr{F}_{2 j}(2 n+1,2 n+2) & =\left\{\psi_{0}(\omega), \psi_{1}(\omega): \omega \in \mathscr{B}_{j}(n, n+1)\right\}, \\
\mathscr{F}_{2 j+1}(2 n+1,2 n+2) & =\left\{\psi_{2}(\omega): \omega \in \mathscr{B}_{j}(n, n+1)\right\} .
\end{aligned}
$$

We have the following properties of the fixed points $\mathscr{F}(2 n+1,2 n+2)$.

Lemma 4.11. For $0 \leq j \leq n$ and any path $\omega \in \mathscr{B}_{j}(n, n+1)$, the following properties hold:

(i) The path $\psi_{0}(\omega)$ contains no odd peaks and odd valleys.

(ii) The path $\psi_{1}(\omega)$ contains a unique odd valley $(1,2 j)$ and no odd peaks.

(iii) The path $\psi_{2}(\omega)$ contains a unique odd peak $(0,2 j+1)$ and no odd valleys.

(iv) $\left|\mathscr{F}_{2 j}(2 n+1,2 n+2)\right|=2\left|\mathscr{B}_{j}(n, n+1)\right|$ and $\left|\mathscr{F}_{2 j+1}(2 n+1,2 n+2)\right|=\left|\mathscr{B}_{j}(n, n+1)\right|$.

It follows that $\operatorname{sump}\left(\psi_{0}(\omega)\right), \operatorname{sump}\left(\psi_{1}(\omega)\right)$ are even and $\operatorname{sump}\left(\psi_{2}(\omega)\right)$ is odd. In fact, $\mathscr{F}(2 n+1,2 n+2)$ consists of all the paths in $\mathscr{B}(2 n+1,2 n+2)$ containing no odd valley $(x, y)$ with $x \geq 2$ and no odd peak $\left(x^{\prime}, y^{\prime}\right)$ with $x^{\prime} \geq 1$.

Now, we construct the involution $\Phi_{3}$ on $\mathscr{B}(2 n+1,2 n+2)$. 
Algorithm 4.12. Given a path $\pi \in \mathscr{B}(2 n+1,2 n+2)$, let $z$ denote the last step of $\pi$. Let $\pi^{\prime}$ be the path obtained from $\pi$ by removing $z$. We consider the following two cases according to the step $z$.

Case 1. $z=N$. Then $\pi^{\prime}$ goes from $(0,0)$ to $(2 n+2,2 n)$. Applying Algorithm 4.3 to the primal factorization of $\pi^{\prime}$, we determine the path $\Phi_{1}\left(\pi^{\prime}\right)$ associated with $\pi^{\prime}$ under the map $\Phi_{1}$. Then the corresponding path $\Phi_{3}(\pi)$ is obtained from $\Phi_{1}\left(\pi^{\prime}\right)$ by appending a north step, i.e., $\Phi_{3}(\pi)=\Phi_{1}\left(\pi^{\prime}\right) N \in \mathscr{B}(2 n+1,2 n+2)$.

Case 2. $z=E$. Then $\pi^{\prime}$ goes from $(0,0)$ to $(2 n+1,2 n+1)$. Applying Algorithm 4.8 to the primal factorization of $\pi^{\prime}$, we determine the path $\Phi_{2}\left(\pi^{\prime}\right)$ associated with $\pi^{\prime}$ under the map $\Phi_{2}$. Then the corresponding path $\Phi_{3}(\pi) \in \mathscr{B}(2 n+1,2 n+2)$ is obtained from $\Phi_{2}\left(\pi^{\prime}\right)$ by appending an east step, i.e., $\Phi_{3}(\pi)=\Phi_{2}\left(\pi^{\prime}\right) E \in \mathscr{B}(2 n+1,2 n+2)$.

Note that the construction of the map $\Phi_{3}$ in Case 1 (Case 2, respectively) of Algorithm 4.12 is similar to the construction of $\Phi_{1}$ by Algorithm 4.3 ( $\Phi_{2}$ by Algorithm 4.8 . respectively). The following property of the map $\Phi_{3}$ can be proved by the same argument as in the proofs of Propositions 4.6 and 4.10 .

Proposition 4.13. For $0 \leq i \leq 2 n+1$, the map $\Phi_{3}$ establishes a refined involution on $\mathscr{B}_{i}(2 n+1,2 n+2)-\mathscr{F}_{i}(2 n+1,2 n+2)$. Moreover, a path $\pi$ is carried to a path $\Phi_{3}(\pi)$ such that $\operatorname{sump}\left(\Phi_{3}(\pi)\right)$ has the opposite parity of $\operatorname{sump}(\pi)$.

Proof of Theorem 1.5(iii).

$$
\begin{aligned}
& \sum_{\sigma \in \mathscr{I}_{4 n+3}(321)}(-1)^{\operatorname{maj}(\sigma)} q^{\operatorname{lead}(\sigma)} \\
= & \sum_{i=0}^{2 n+1}\left(\sum_{\pi \in \mathscr{B}_{i}(2 n+1,2 n+2)}(-1)^{\operatorname{sump}(\pi)}\right) q^{i+1} \\
= & \sum_{j=0}^{n}\left(\sum_{\pi \in \mathscr{F}_{2 j}(2 n+1,2 n+2)} q^{2 j+1}-\sum_{\pi \in \mathscr{F}_{2 j+1}(2 n+1,2 n+2)} q^{2 j+2}\right) \\
= & \sum_{j=0}^{n} 2\left|\mathscr{B}_{j}(n, n+1)\right| q^{2 j+1}-\sum_{j=0}^{n}\left|\mathscr{B}_{j}(n, n+1)\right| q^{2 j+2} \\
= & \left(\frac{2}{q}-1\right) \sum_{\sigma \in \mathscr{I}_{2 n+1}(321)} q^{2 \cdot \operatorname{lead}(\sigma)} .
\end{aligned}
$$

4.4. The case $\Phi_{4}: \mathscr{B}(2 n, 2 n+1) \rightarrow \mathscr{B}(2 n, 2 n+1)$

To prove Theorem 1.5 (iv), we shall establish a sump-parity-reversing involution $\Phi_{4}: \mathscr{B}_{i}(2 n$, $2 n+1) \rightarrow \mathscr{B}_{i}(2 n, 2 n+1)$ for $0 \leq i \leq 2 n$. Let $\mathscr{F}(2 n, 2 n+1) \subseteq \mathscr{B}(2 n, 2 n+1)$ be the set 
of fixed points of the map $\Phi_{4}$. For $0 \leq i \leq 2 n$, we define

$$
\mathscr{F}_{i}(2 n, 2 n+1)=\mathscr{F}(2 n, 2 n+1) \cap \mathscr{B}_{i}(2 n, 2 n+1) .
$$

The set $\mathscr{F}(2 n, 2 n+1)$ can be constructed from $\mathscr{B}(n, n+1)$ as follows. For each path $\omega \in \mathscr{B}_{j}(n, n+1)(0 \leq j \leq n)$, we form a path $\gamma(\omega)$ by duplicating every step of $\omega$. We consider the following two cases according to the last step $z$ of $\omega$ :

- $z=$ E. Then the last two steps of $\gamma(\omega)$ are east steps. Let $\varphi_{0}(\omega)$ be the path obtained from $\gamma(\omega)$ by removing the last step.

- $z=\mathrm{N}$. Then the last two steps of $\gamma(\omega)$ are north steps. Factorize $\gamma(\omega)$ as $\mathrm{N}^{2 j} \mathrm{EE} \beta \mathrm{NN}$ and let $\varphi_{1}(\omega)\left(\varphi_{2}(\omega)\right.$, respectively) be the path obtained from $\gamma(\omega)$ by inserting a north step after (before, respectively) the first east step and then removing the second east step and the last step, i.e.,

$$
\varphi_{1}(\omega)=\mathrm{N}^{2 j} \mathrm{EN} \beta \mathrm{N}, \quad \varphi_{2}(\omega)=\mathrm{N}^{2 j+1} \mathrm{E} \beta \mathrm{N}
$$

For example, for $\omega_{1}=$ NEENENE $\in \mathscr{B}_{1}(3,4)$, the path $\varphi_{0}\left(\omega_{1}\right)$ is shown as the left-hand side of Figure 4.6. For $\omega_{2}=$ EENENEN $\in \mathscr{B}_{0}(3,4)$, the paths $\varphi_{1}\left(\omega_{2}\right), \varphi_{2}\left(\omega_{2}\right)$ are shown as the right-hand side of Figure 4.6 .
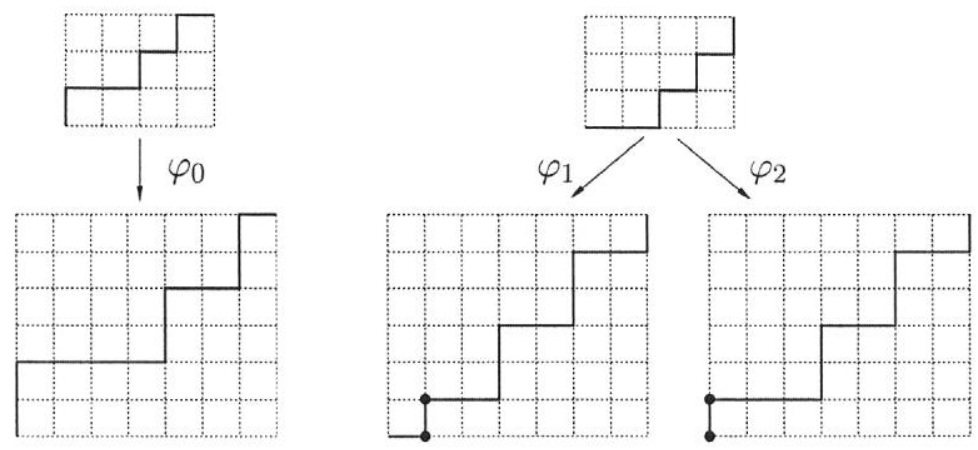

Figure 4.6: Examples of the maps $\varphi_{0}, \varphi_{1}$ and $\varphi_{2}$

We define the refinement of the set $\mathscr{F}(2 n, 2 n+1)$. For $0 \leq j \leq n$, let $\mathscr{F}_{2 j}(2 n, 2 n+1)=$ $\mathscr{F}_{2 j}^{E}(2 n, 2 n+1) \cup \mathscr{F}_{2 j}^{N}(2 n, 2 n+1)$, where

$$
\begin{aligned}
\mathscr{F}_{2 j}^{E}(2 n, 2 n+1) & =\left\{\varphi_{0}(\omega): \omega \in \mathscr{B}_{j}(n, n+1) \text { ends with an east step }\right\} \\
\mathscr{F}_{2 j}^{N}(2 n, 2 n+1) & =\left\{\varphi_{1}(\omega): \omega \in \mathscr{B}_{j}(n, n+1) \text { ends with a north step }\right\} \\
\mathscr{F}_{2 j+1}(2 n, 2 n+1) & =\left\{\varphi_{2}(\omega): \omega \in \mathscr{B}_{j}(n, n+1) \text { ends with a north step }\right\} .
\end{aligned}
$$

We have the following properties of the fixed points $\mathscr{F}(2 n+1,2 n+2)$. 
Lemma 4.14. For $0 \leq j \leq n$ and any path $\omega \in \mathscr{B}_{j}(n, n+1)$, the following properties hold:

(i) The path $\varphi_{0}(\omega)$ contains no odd peaks and odd valleys.

(ii) The path $\varphi_{1}(\omega)$ contains a unique odd valley $(1,2 j)$ and no odd peaks.

(iii) The path $\varphi_{2}(\omega)$ contains a unique odd peak $(0,2 j+1)$ and no odd valleys.

(iv) $\left|\mathscr{F}_{2 j}(2 n+1,2 n+2)\right|=\left|\mathscr{B}_{j}(n, n+1)\right|$.

(v) $\left|\mathscr{F}_{2 j+1}(2 n+1,2 n+2)\right|=\left|\mathscr{B}_{j}(n, n+1)\right|-\left|\mathscr{B}_{j}(n, n)\right|$.

It follows that $\operatorname{sump}\left(\varphi_{0}(\omega)\right), \operatorname{sump}\left(\varphi_{1}(\omega)\right)$ are even and $\operatorname{sump}\left(\varphi_{2}(\omega)\right)$ is odd. Now, we construct the involution $\Phi_{4}$ on $\mathscr{B}(2 n, 2 n+1)$.

Algorithm 4.15. Given a path $\pi \in \mathscr{B}(2 n, 2 n+1)$, let $z$ denote the last step of $\pi$. Let $\pi^{\prime}$ be the path obtained from $\pi$ by removing $z$. We consider the following two cases according to the step $z$.

Case 1. $z=E$. Then $\pi^{\prime}$ goes from $(0,0)$ to $(2 n, 2 n)$. By Algorithm 4.3, we determine the path $\Phi_{1}\left(\pi^{\prime}\right) \in \mathscr{B}(2 n, 2 n)$ associated with $\pi^{\prime}$ under the map $\Phi_{1}$. Then the corresponding path $\Phi_{4}(\pi)$ is obtained from $\Phi_{1}\left(\pi^{\prime}\right)$ by appending an east step in the end, i.e., $\Phi_{4}(\pi)=$ $\Phi_{1}\left(\pi^{\prime}\right) E$.

Case 2. $z=N$. Then $\pi^{\prime}$ goes from $(0,0)$ to $(2 n+1,2 n-1)$. By the same method as in Algorithm 4.8, we determine the path $\Phi_{2}\left(\pi^{\prime}\right) \in \mathscr{B}(2 n-1,2 n+1)$ associated with $\pi^{\prime}$ under the map $\Phi_{2}$. Then the corresponding path $\Phi_{4}(\pi) \in \mathscr{B}(2 n, 2 n+1)$ is obtained from $\Phi_{2}\left(\pi^{\prime}\right)$ by appending a north step in the end, i.e., $\Phi_{4}(\pi)=\Phi_{2}\left(\pi^{\prime}\right) N$.

The following property of the map $\Phi_{4}$ can be proved by the same argument as in the proofs of Propositions 4.6 and 4.10 since the construction of $\Phi_{4}$ in Case 1 (Case 2, respectively) of Algorithm 4.15 is similar to the construction of $\Phi_{1}\left(\Phi_{2}\right.$, respectively).

Proposition 4.16. For $0 \leq i \leq 2 n$, the map $\Phi_{4}$ establishes a refined involution on $\mathscr{B}_{i}(2 n, 2 n+1)-\mathscr{F}_{i}(2 n, 2 n+1)$. Moreover, a path $\pi$ is carried to a path $\Phi_{4}(\pi)$ such that $\operatorname{sump}\left(\Phi_{4}(\pi)\right)$ has the opposite parity of $\operatorname{sump}(\pi)$.

Proof of Theorem 1.5(iv).

$$
\begin{aligned}
& \sum_{\sigma \in \mathscr{I}_{4 n+1}(321)}(-1)^{\operatorname{maj}(\sigma)} q^{\operatorname{lead}(\sigma)} \\
= & \sum_{i=0}^{2 n}\left(\sum_{\pi \in \mathscr{B}_{i}(2 n, 2 n+1)}(-1)^{\operatorname{sump}(\pi)}\right) q^{i+1}
\end{aligned}
$$




$$
\begin{aligned}
& =\sum_{j=0}^{n}\left(\sum_{\pi \in \mathscr{F}_{2 j}(2 n, 2 n+1)} q^{2 j+1}-\sum_{\pi \in \mathscr{F}_{2 j+1}(2 n, 2 n+1)} q^{2 j+2}\right) \\
& =\sum_{j=0}^{n}\left(\left|\mathscr{B}_{j}(n, n+1)\right|-\left|\mathscr{B}_{j}(n, n+1)\right| q+\left|\mathscr{B}_{j}(n, n)\right| q\right) q^{2 j+1} \\
& =\left(\frac{1}{q}-1\right) \sum_{\sigma \in \mathscr{I}_{2 n+1}(321)} q^{2 \cdot \operatorname{lead}(\sigma)}+\sum_{\sigma \in \mathscr{I}_{2 n}(321)} q^{2 \cdot \operatorname{lead}(\sigma)} .
\end{aligned}
$$

\section{Analogous results for 123 -avoiding involutions}

It is known that by the Robinson-Schensted-Knuth (RSK) algorithm an involution $\sigma \in$ $\mathscr{I}_{n}(321)\left(\mathscr{I}_{n}(123)\right.$, respectively) is associated with a pair $(Q, Q)$ of identical $n$-cell standard Young tableaux with at most two rows (columns, respectively). Let $Q^{\mathrm{T}}$ be the transpose of $Q$ and let $\sigma^{\mathrm{T}}$ be the preimage of the pair $\left(Q^{\mathrm{T}}, Q^{\mathrm{T}}\right)$ under the RSK correspondence. Then $\sigma \leftrightarrow \sigma^{\mathrm{T}}$ is a bijection between $\mathscr{I}_{n}(321)$ and $\mathscr{I}_{n}(123)$.

Lemma 5.1. We have

$$
\operatorname{Des}\left(\sigma^{\mathrm{T}}\right)=\{i: i \notin \operatorname{Des}(\sigma), 1 \leq i \leq n-1\}
$$

Proof. It is known that a descent $\sigma_{i}>\sigma_{i+1}$ in $\sigma$ is translated to the 'descent' of the recording tableau $Q$ that the entry $i+1$ is in a row lower than the row of $i$. For $1 \leq i \leq n-1$, if $i \in \operatorname{Des}(\sigma)$ then $i(i+1$, respectively) is in the first (second, respectively) row in $Q$. Then $i+1$ is either in the same column as $i$ or in a column to the left of the column of $i$ in $Q$. Then $i+1$ is not in a lower row than the row of $i$ in $Q^{\mathrm{T}}$. Hence $i \notin \operatorname{Des}\left(\sigma^{\mathrm{T}}\right)$.

Otherwise, $i \notin \operatorname{Des}(\sigma)$. Then $i+1$ is not in a row lower than the row of $i$ in $Q$. The element $i$ is in the first row or the second row. In either case, the element $i+1$ is in a column to the right of the column of $i$. Then $i+1$ is in row lower than the row of $i$ in $Q^{\mathrm{T}}$. Hence $i \in \operatorname{Des}\left(\sigma^{\mathrm{T}}\right)$.

We obtain the joint distribution of major index and descent number for 123-avoiding involutions.

Corollary 5.2. We have

$$
\sum_{\substack{\sigma \in \mathscr{I}_{n}(123) \\
\operatorname{des}(\sigma)=n-1-k}} q^{\operatorname{maj}(\sigma)}=q^{\left(\begin{array}{c}
n \\
2
\end{array}\right)+k^{2}-n k}\left[\begin{array}{c}
\lceil n / 2\rceil \\
k
\end{array}\right]_{q}\left[\begin{array}{c}
\lfloor n / 2\rfloor \\
k
\end{array}\right]_{q}
$$

Proof. Substituting $q^{-1}$ for $q$ in Theorem 1.4(i), we have

$$
\sum_{\substack{\sigma \in \mathscr{I}_{n}(321) \\
\operatorname{des}(\sigma)=k}} q^{-\operatorname{maj}(\sigma)}=q^{-k^{2}}\left[\begin{array}{c}
\lceil n / 2\rceil \\
k
\end{array}\right]_{q^{-1}}\left[\begin{array}{c}
\lfloor n / 2\rfloor \\
k
\end{array}\right]_{q^{-1}}=q^{k^{2}-k n}\left[\begin{array}{c}
\lceil n / 2\rceil \\
k
\end{array}\right]_{q}\left[\begin{array}{c}
\lfloor n / 2\rfloor \\
k
\end{array}\right]_{q}
$$


By Lemma 5.1, $\operatorname{des}(\sigma)=n-1-\operatorname{des}\left(\sigma^{\mathrm{T}}\right)$ and $\operatorname{maj}(\sigma)=\left(\begin{array}{c}n \\ 2\end{array}\right)-\operatorname{maj}\left(\sigma^{\mathrm{T}}\right)$ for any $\sigma \in \mathscr{I}_{n}(123)$. Thus we have

$$
\sum_{\substack{\sigma \in \mathscr{I}_{n}(123) \\
\operatorname{des}(\sigma)=n-1-k}} q^{\operatorname{maj}(\sigma)}=\sum_{\substack{\sigma^{\mathrm{T}} \in \mathscr{I}_{n}(321) \\
\operatorname{des}\left(\sigma^{\mathrm{T}}\right)=k}} q^{\left(\begin{array}{l}
n \\
2
\end{array}\right)-\operatorname{maj}\left(\sigma^{\mathrm{T}}\right)}=q^{\left(\begin{array}{l}
n \\
2
\end{array}\right)+k^{2}-n k}\left[\begin{array}{c}
\lceil n / 2\rceil \\
k
\end{array}\right]_{q}\left[\begin{array}{c}
\lfloor n / 2\rfloor \\
k
\end{array}\right]_{q},
$$

as required.

With the bijection $\sigma \leftrightarrow \sigma^{\mathrm{T}}$ between $\mathscr{I}_{n}(321)$ and $\mathscr{I}_{n}(123)$, we can prove affirmatively Conjecture 1.3. In fact, this result is essentially equivalent to Theorem 1.2 .

Theorem 5.3. For all $n \geq 1$, we have

(i) $\sum_{\sigma \in \mathscr{I}_{4 n}(123)}(-1)^{\operatorname{maj}(\sigma)} q^{\operatorname{des}(\sigma)}=q \sum_{\sigma \in \mathscr{I}_{2 n}(123)} q^{2 \cdot \operatorname{des}(\sigma)}$,

(ii) $\sum_{\sigma \in \mathscr{I}_{4 n+2}(123)}(-1)^{\operatorname{maj}(\sigma)} q^{\operatorname{des}(\sigma)}=(1-q) q^{2} \sum_{\sigma \in \mathscr{I}_{2 n}(123)} q^{2 \cdot \operatorname{des}(\sigma)}$,

(iii) $\sum_{\sigma \in \mathscr{I}_{2 n+1}(123)}(-1)^{\operatorname{maj}(\sigma)} q^{\operatorname{des}(\sigma)}=(-1)^{n} q^{2} \sum_{\sigma \in \mathscr{I}_{n}(123)} q^{2 \cdot \operatorname{des}(\sigma)}$.

Proof. Note that $\operatorname{des}(\sigma)=n-1-\operatorname{des}\left(\sigma^{\mathrm{T}}\right)$ and $\operatorname{maj}(\sigma)=\left(\begin{array}{l}n \\ 2\end{array}\right)-\operatorname{maj}\left(\sigma^{\mathrm{T}}\right)$ for any $\sigma \in$ $\mathscr{I}_{n}(123)$. Making use of the identity in Theorem $1.2(\mathrm{i})$, we have

$$
\begin{aligned}
\sum_{\sigma \in \mathscr{I}_{4 n}(123)}(-1)^{\operatorname{maj}(\sigma)} q^{\operatorname{des}(\sigma)} & =\sum_{\sigma^{\mathrm{T}} \in \mathscr{I}_{4 n}(321)}(-1)^{\left(\begin{array}{c}
4 n \\
2
\end{array}\right)-\operatorname{maj}\left(\sigma^{\mathrm{T}}\right)} q^{4 n-1-\operatorname{des}\left(\sigma^{\mathrm{T}}\right)} \\
& \left.=q \sum_{\sigma^{\mathrm{T}} \in \mathscr{I}_{2 n}(321)} q^{2\left(2 n-1-\operatorname{des}\left(\sigma^{\mathrm{T}}\right)\right)} \quad \text { (by Theorem } 1.2(\mathrm{i})\right) \\
& =q \sum_{\sigma \in \mathscr{I}_{2 n}(123)} q^{2 \cdot \operatorname{des}(\sigma)}
\end{aligned}
$$

The assertion (i) follows. Making use of the identities in (ii) and (iii) of Theorem 1.2 , the assertions (ii) and (iii) can be proved straightforward in the same manner.

\section{Acknowledgments}

The authors thank the referees for carefully reading the manuscript and providing helpful suggestions. This research is partially supported by MOST grant 105-2115-M-153-002MY2 (T.-S. Fu) and MOST postdoctoral fellowship 106-2811-M-003-011 (H.-C. Hsu). 


\section{References}

[1] R. M. Adin and Y. Roichman, Equidistribution and sign-balance on 321-avoiding permutations, Sém. Lothar. Combin. 51 (2004) Art. B51d, 14 pp.

[2] M. Barnabei, F. Bonetti, S. Elizalde and M. Silimbani, Descent sets on 321-avoiding involutions and hook decompositions of partitions, J. Combin. Theory Ser. A 128 (2014), 132-148.

[3] E. Deutsch, A. Robertson and D. Saracino, Refined restricted involutions, European J. Combin. 28 (2007), no. 1, 481-498.

[4] S.-P. Eu, T.-S. Fu, Y.-J. Pan and C.-T. Ting, Sign-balance identities of AdinRoichman type on 321-avoiding alternating permutations, Discrete Math. 312 (2012), no. $15,2228-2237$.

[5] _ Two refined major-balance identities on 321-avoiding involutions, European J. Combin. 49 (2015), 250-264.

[6] A. Reifegerste, Refined sign-balance on 321-avoiding permutations, European J. Combin. 26 (2005), no. 6, 1009-1018.

[7] R. Simion and F. W. Schmidt, Restricted permutations, European J. Combin. 6 (1985), no. 4, 383-406.

[8] R. P. Stanley, Enumerative Combinatorics, Volume 2, Cambridge Studies in Advanced Mathematics 62, Cambridge University Press, Cambridge, 1999.

Tung-Shan Fu

Department of Applied Mathematics, National Pingtung University, Pingtung 90003,

Taiwan

E-mail address: tsfu@mail.nptu.edu.tw

Hsiang-Chun Hsu

Department of Mathematics, National Taiwan Normal University, Taipei 11677, Taiwan E-mail address: hchsu0222@gmail.com

Hsin-Chieh Liao

Department of Mathematics, National Taiwan Normal University, Taipei 11677, Taiwan E-mail address: jeffliao1@gmail.com 